\title{
Técnicas quirúrgicas complejas en el tratamiento con implantes oseointegrados del maxilar superior. Un seguimiento clínico de dos años
}

\author{
PÉREZ PÉREZ O* \\ VELASCO ORTEGA E** \\ GONZÁLEZ OLIVARES LL*** \\ GARCÍA MÉNDEZ A**** \\ RODRÍGUEZ CALZADILLA O*****
}

\begin{abstract}
Pérez Pérez O, Velasco Ortega E, González Olivares Ll, García Méndez A, Rodríguez Calzadilla O. Técnicas quirúrgicas complejas en el tratamiento con implantes oseointegrados del maxilar superior. Un seguimiento clínico de 2 años. Av Periodon Implantol. 2007; 19, Suppl: 25-34.
\end{abstract}

\section{RESUIMEN}

Introducción. El objetivo del presente estudio era valorar los resultados clínicos del tratamiento con implantes oseointegrados en el maxilar superior con técnicas quirúrgicas complejas.

Pacientes y métodos. 51 pacientes fueron tratados con 93 implantes Microdent ${ }^{\circledR}$ con superficie con chorreado de arena y grabada con ácidos para la rehabilitación del maxilar superior mediante la técnica de elevación de seno, expansión ósea e injertos óseos. Los implantes fueron cargados después de un periodo de cicatrización de 6 meses y fueron evaluados más de 2 años.

Resultados. 37 pacientes $(72,5 \%)$ fueron tratados mediante elevación del seno maxilar. 8 pacientes $(15,6 \%)$ fueron tratados mediante la técnica de expansión ósea y 6 pacientes $(11,7 \%)$ con injertos óseos. El 83,3\% de los implantes fueron insertados en el sector posterior maxilar. Los hallazgos clínicos indican una supervivencia y éxito de los implantes del $96,8 \%$, ya que 3 implantes se perdieron durante el periodo de cicatrización.

Conclusiones. Este estudio indica que el tratamiento mediante técnicas quirúrgicas complejas del maxilar superior con implantes oseointegrados constituye un tratamiento odontológico con una elevada tasa de éxito.

\section{PALABRAS CLAVE}

Implantes orales, elevación del seno maxilar, expansión ósea, injertos óseos, implantología oral, maxilar superior.

\section{Aceptado para publicación: 2005}

Publacado en Av Periodon Implantol. 2006; 18, 1: 10-19.

\footnotetext{
* Profesor de Cirugía Oral y Máxilofacial. Facultad de Estomatología. Universidad de La Habana. Cuba.

** Profesor Titular de Odontología Integrada de Adultos y Gerodontología. Facultad de Odontología. Universidad de Sevilla.

*** Estomatóloga General. Policlínica Salvador Allende. La Habana. Cuba

**** Profesor Asociado de Odontología Integrada de Adultos. Facultad de Odontología. Universidad de Sevilla.

***** Profesor de Cirugía Máxilofacial. Hospital Aleida Fernández. La Habana. Cuba.
} 


\section{INTRODUCCIÓN}

La rehabilitación protésica mediante implantes oseointegrados se ha ido incorporando a la práctica odontológica cotidiana como alternativa de tratamiento odontológico desde que Branemark y sus colaboradores presentara su experiencia con el uso de implantes en pacientes desdentados ${ }^{1}$. Desde un punto de vista, sobre todo quirúrgico, el maxilar superior supone un reto importante para el tratamiento con implantes oseointegrados por su anatomía y por la frecuente escasa disponibilidad y calidad de hueso debido a la pérdida prematura de su dentición natural ${ }^{2}$.

La atrofia progresiva del maxilar superior está relacionada con factores mecánicos, inflamatorios, sistémicos o metabólicos. La pérdida dental por exodoncias o traumatismos provoca un proceso de remodelación alveolar casi siempre en el sentido de la de atrofia vertical y vestíbulo-palatina que se incrementa con la acción de las prótesis removibles. Además, la neumatización del seno maxilar disminuye notablemente la cantidad de hueso disponible, que unido a la pobre calidad ósea del maxilar superior con un hueso muy esponjoso y una cortical muy delgada (que puedo incluso desaparecer) dificulta la inserción de los implantes orales ${ }^{3-4}$.

Además, los implantes que se insertan en el maxilar superior deben estar situados en posiciones adecuadas y mantener inclinaciones favorables para el posterior diseño prostodóncico, por lo que las bases óseas deben tener las dimensiones adecuadas para soportar las fijaciones implantológicas con garantías de éxito y deben mantener unas relaciones esqueléticas favorables que favorezca la realización de prótesis estéticas y funcionales ${ }^{5-6}$.

Se han desarrollado diversas técnicas quirúrgicas para restaurar el volumen óseo necesario para la colocación de los implantes reconstruyendo tridimensionalmente la cresta alveolar mediante injertos fijados rígidamente al hueso maxilar, precedido por una adecuada valoración protésica-quirúrgica que en el caso del maxilar superior anterior son los injertos aposicionales o en onlay, los interposicionales o inlays, a los que en los últimos tiempos se ha unido la distracción ósea ${ }^{7-9}$.

En los segmentos posteriores donde la estética es menos importante, se prioriza la necesidad de insertar implantes lo suficientemente largos y gruesos que incrementen el contacto hueso- superficie del implante y permitan soportar adecuadamente las fuerzas masticatorias a ese nivel. En este sentido, se ha descri- to la colocación de implantes con fijación en la apófisis pterigoides y en el cuerpo del hueso cigomático, la inclinación de los implantes aprovechando el arbotante que separa el seno maxilar de la fosa nasal, y la técnica mas universalmente aceptada que es la elevación del seno maxilar ${ }^{10-13}$. Se ha reservado la osteotomía de Le Fort I e injertos en sandwich para los casos de atrofia total ${ }^{14}$. Una técnica menos compleja y traumática, también muy utilizada es el aumento del ancho crestal mediante la utilización de expansores que en muchos casos permite mejorar notablemente las dimensiones horizontales para la colocación de implantes ${ }^{15}$.

El objetivo del presente estudio era evaluar la terapéutica con implantes oseointegrados en la rehabilitación del maxilar superior en zonas tratadas con técnicas quirúrgica complejas óseas.

\section{PACIENTES Y MÉTODOS}

El presente estudio fue realizado por profesores de las unidades docentes de Cirugía Oral y Maxilofacial de la Facultad de Estomatología de la Universidad de La Habana (Cuba) y de Odontología Integrada de Adultos de la Facultad de Odontología de la Universidad de Sevilla.

Previamente a la realización del estudio, aquellos pacientes que padecían trastornos sistémicos graves que podían comprometer la oseointegración fueron excluidos del estudio ${ }^{16}$. Los pacientes seleccionados eran adultos, de ambos sexos. Todos los pacientes presentaban una menor disponibilidad de hueso en el maxilar superior que indicaba la necesidad de realizar una técnica quirúrgica compleja para la inserción de los implantes.

Todos los pacientes seleccionados en el estudio fueron informados del protocolo del tratamiento con implantes, de los aspectos quirúrgicos (diversas técnicas quirúrgicas del maxilar superior) y prostodóncicos, temporalización del tratamiento y seguimiento, así como de la posibilidad de la existencia de complicaciones y pérdida de implantes. Así mismo, todos los pacientes autorizaron el tratamiento implantológico mediante un consentimiento informado. Antes del tratamiento, todos los pacientes fueron evaluados radiológicamente, con una ortopantomografía. En los casos más comprometidos desde un punto de vista óseo, se realizó una tomografía axial computarizada (TAC).

Los criterios de éxito y supervivencia de los implantes fueron los recomendados por van Steenberghe et 
al ${ }^{17}$. En este sentido, la supervivencia fue definida como la proporción de implantes permanentes en su localización original aunque no tengan valor clínico o cause efectos adversos. Los criterios de éxito de los implantes se expresan en la Tabla 1.

\section{Cirugía}

Una hora antes de la cirugía, los pacientes comenzaron un régimen antibiótico preventivo (amoxicilina + ácido clavulánico) durante una semana. Así mismo, todos los pacientes fueron tratados durante l semana con ibuprofeno. Todos los pacientes recibieron anestesia local. A la semana se retiraron las suturas y a todos los pacientes se les recomendó el enjuague diario con clorhexidina durante los primeros 30 días.

Todos los implantes insertados fueron Microdent ${ }^{\circledR}$ (Microdent, Barcelona, España) roscados de superficie con chorreado de arena y grabado ácido, y todos fueron estables después de la inserción.

En los casos de elevación del seno maxilar se utilizó como biomaterial de relleno mineral de hueso bovino, Bio-Oss $^{\circledR}$ (Geistlich Pharma AG, Wolhusen, Suiza). Cuan-

\section{TABLA 1.- LOS CRITERIOS DE EXITO EN EL TRATAMIENTO CON IMPLANTES (Van Steenberghe et al ${ }^{17}$ )}

1. El implante no provoca ninguna reacción alérgica, tóxica o infecciosa de carácter local o sistémico.

2. El implante ofrece soporte para una prótesis funcional.

3. El implante no muestra signos de fractura o incurvación.

4. El implante no muestra ninguna movilidad cuando es explorado manual o electrónicamente.

5. El implante no muestra ningún signo de radiolucidez mediante una radiografía intraoral.

6. La pérdida marginal de hueso ( $R x$ intraoral) y/o la pérdida de inserción (profundidad de sondaje + recesión) no deben perjudicar la función de anclaje del implante o causar molestias para el paciente durante 20 años. do la altura del hueso era menor de $4 \mathrm{~mm}$, se utilizó además hueso del mismo paciente (ej. mentón, rama mandibular, tuberosidad del maxilar).

\section{Prostodoncia}

A los 6 meses, después de la inserción de los implantes, se realizó la carga funcional de los implantes mediante la colocación de las correspondientes prótesis implantosoportadas. El tiempo transcurrido de seguimiento clínico desde la carga funcional de los implantes fue al menos de 24 meses.

\section{ANÁLISIS ESTAdístico}

Se realizó una estadística descriptiva de los hallazgos clínicos del estudio, con referencia a las variables demográficas de los pacientes, las diversas técnicas quirúrgicas orales, la inserción, supervivencia y pérdida de los implantes así como del seguimiento clínico de las restauraciones prostodóncicas realizadas.

\section{RESULTADOS}

De los 51 pacientes tratados, 38 pacientes eran hombres $(74,5 \%)$ y 13 eran mujeres (25,5\%). La edad media era de 38 años (rango: 18-71 años). Con respecto al consumo de tabaco, 40 pacientes no fumaban $(78,4 \%)$ mientras que 11 eran fumadores $(21,6 \%)$.

Se insertaron un total de 93 implantes en el maxilar superior, de los correspondientes 51 pacientes (Tabla 2), lo que representa una media de 1,8 implantes por paciente. 74 implantes fueron insertados en 34 pacien-

\section{TABLA 2.- DISTRIBUCION DE LASS TÉCNICAS QUIRÚRGICAS COMPLEJAS}

\begin{tabular}{|lcr|}
\hline Técnicas & Pacientes & Total \\
\hline Injerto & 6 & 11 \\
Expansión ósea & 8 & 8 \\
Elevación seno maxilar & 37 & 74 \\
\hline Total & 51 & 93 \\
\hline
\end{tabular}




\section{AVANCES}

Volumen 19 - Suppl. - Febrero 2007

tes mediante la elevación del seno maxilar, 11 implantes fueron insertados en 6 pacientes realizando injertos óseos y 8 implantes se insertaron mediante expansores en 8 pacientes (Figuras 1-5).

Los diversos tipos de implantes según su longitud y diámetro se exponen en las Tablas 3 y 4 . La longitud variaba entre 10 y $14 \mathrm{~mm}$, mientras que el diámetro de los implantes variaba de $3 \mathrm{~mm}$ a 4,2 $\mathrm{mm}$ (Tablas 3 y 4 ). El 83,8\% de los implantes fueron insertados en el sector posterior, mientras que el $16,2 \%$ en el sector anterior del maxilar superior.

En 3 implantes (3,2\%) hubo complicaciones, al presentar movilidad a la exploración clínica durante la fase de cicatrización libre de carga funcional y fueron ex-

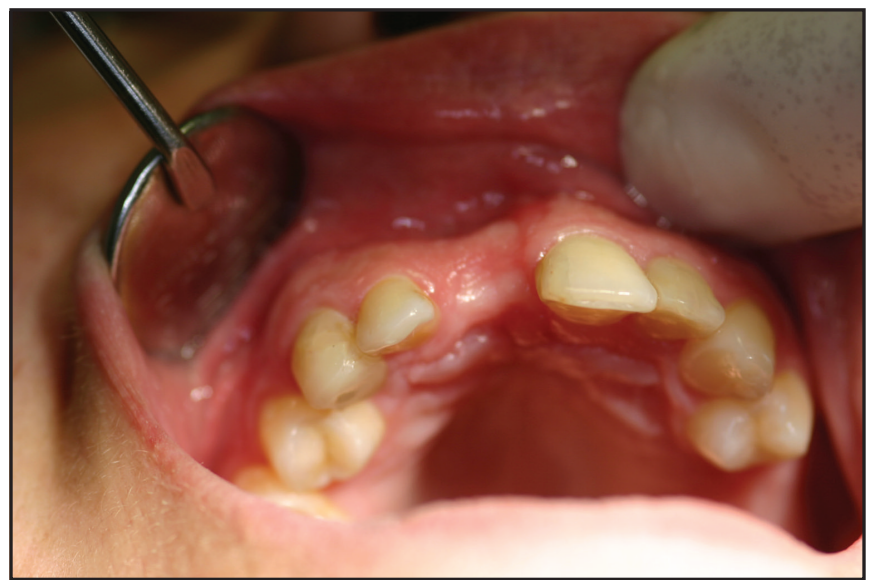

Fig. 1: Aspecto clínico del paciente con pérdida dental unitaria.

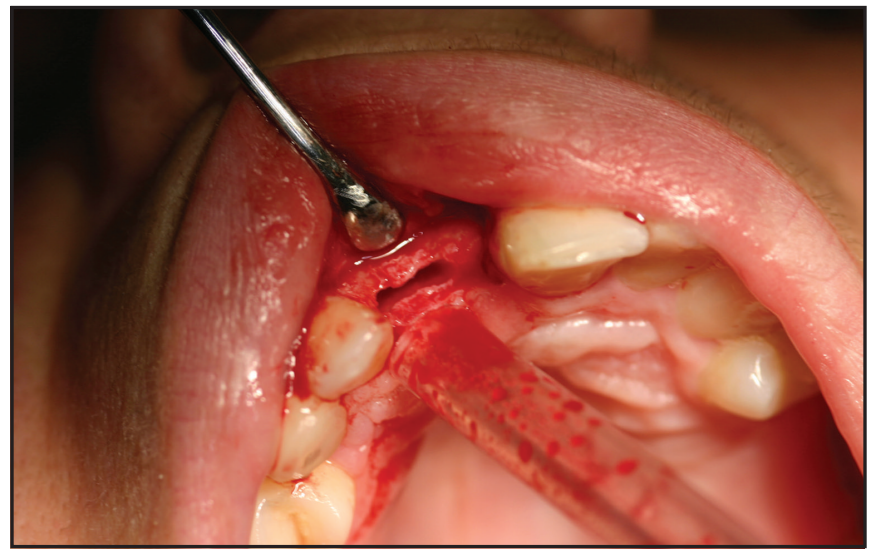

Fig. 2: Primera cirugía o fase quirúrgica. Osteotomía de la cresta alveolar.

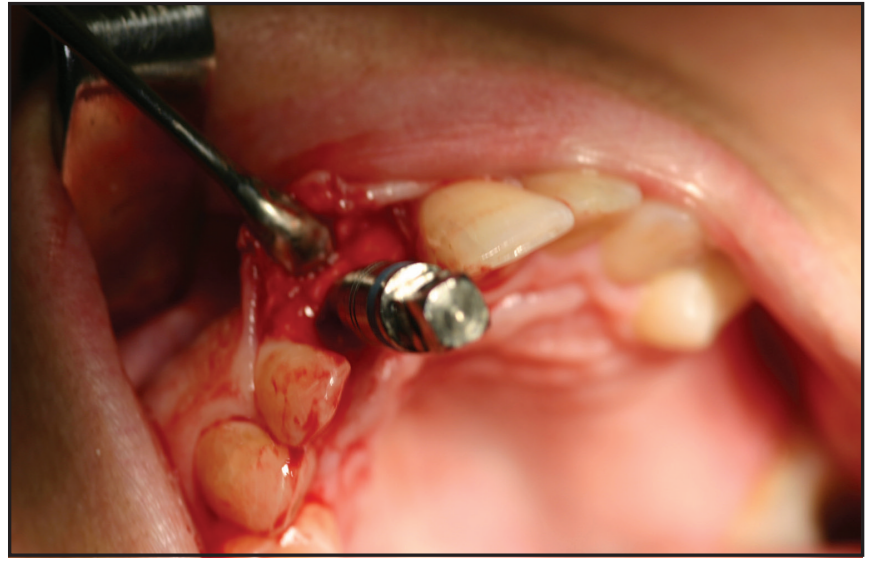

Fig. 3: Primera cirugía o fase quirúrgica. Inserción progresiva de los expansores.

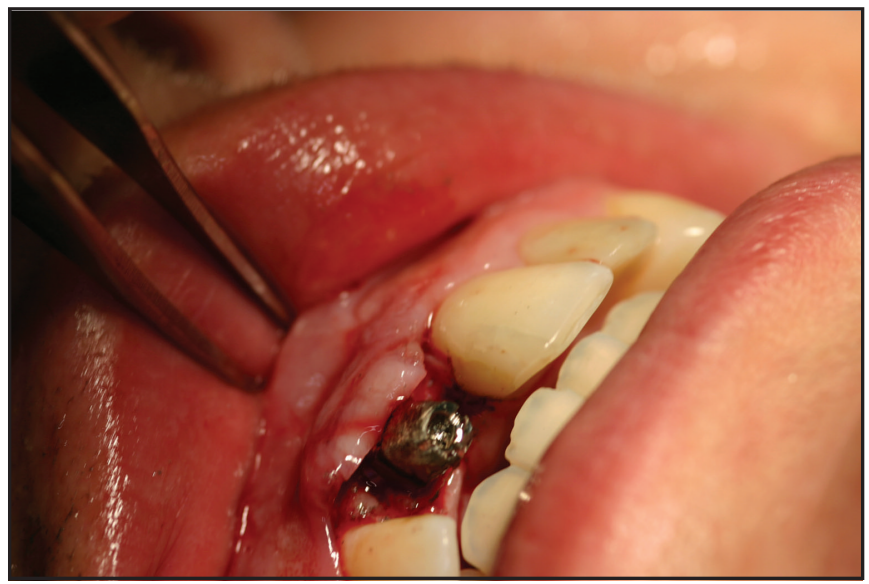

Fig.4: Segunda cirugía a los 6 meses. Colocación del pilar protésico.

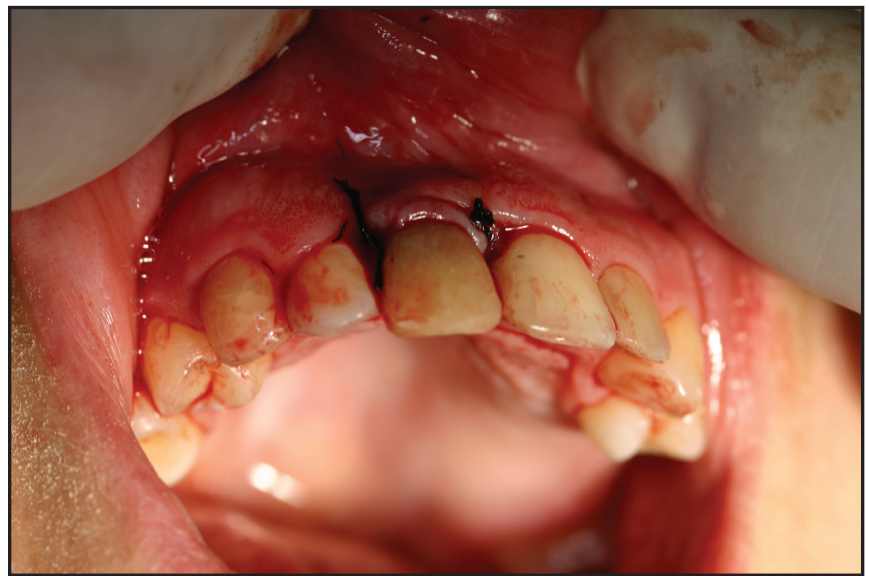

Fig. 5: Colocación de la corona provisional. 


\begin{tabular}{|lcccccc|}
\hline \multicolumn{7}{|c|}{ TABLA 3.- DISTRIBUCIÓN DE LA LONGITUD DE LOS IMPLANTES SEGÚN } \\
LAS TÉCNICAS QUIRÚRGICAS COIMPLEJAS \\
\hline Técnicas & $\mathbf{1 0} \mathbf{~ m m}$ & $\mathbf{1 1 , 5} \mathbf{~ m m}$ & $\mathbf{1 2} \mathbf{~ m m}$ & $\mathbf{1 3} \mathbf{~ m m}$ & $\mathbf{1 4} \mathbf{~ m m}$ & Total \\
\hline Injerto & 2 & 1 & 1 & 4 & 3 & 11 \\
Expansión ósea & 4 & 2 & 2 & - & - & 8 \\
Elevación seno maxilar & 9 & 3 & 38 & 11 & 13 & 74 \\
\hline Total & 15 & 6 & 41 & 15 & 16 & 93 \\
\hline
\end{tabular}

traídos; 2 implantes fracasaron en 2 pacientes del sexo femenino; el tercero, en un paciente varón. De los 3 pacientes en que fracasaron los implantes 2 eran fumadores. Los fracasos se localizaron en los sectores posteriores en los 3 pacientes.

El tiempo medio de seguimiento clínico desde la carga funcional de los implantes fue de 30,4 meses. Ningún implante se ha perdido después de la carga funcional prostodóncica durante el periodo de seguimiento clínico.

\section{DISCUSIÓN}

El presente estudio valora los resultados clínicos del tratamiento con implantes oseointegrados en el maxilar superior con una insuficiente cantidad o disponibilidad de hueso. En este sentido, la fase quirúrgica del tratamiento no se realizó mediante una técnica quirúrgica básica, sino que fue necesario en todos los casos la realización de técnicas más complejas que incrementaran de alguna forma el volumen óseo (ej. injertos, elevación de seno maxilar) o mejoraran la posterior inserción de los implantes (ej. expansión ósea) ${ }^{2,6-1}$.

Algunos requisitos son imprescindibles para el éxito predecible de estas técnicas quirúrgicas complejas en el tratamiento con implantes del maxilar superior. El primer requisito se corresponde con un diagnóstico integral y preciso del paciente. El paciente debe ser evaluado desde un punto de vista anatómico de sus estructuras óseas mediante una ortopantomografía y en los casos necesarios con una tomografía computarizada. El análisis de los modelos de estudios ayudarán a diseñar las futuras restauraciones prostodoncias implantosoportadas. La valoración global indicará la selección de los pacientes (ej. factores de riesgo como el tabaco) y de la técnica quirúrgica más necesaria y recomendable, así como el número, tipo y localización de los implantes ${ }^{16,18-19}$.

\begin{tabular}{|c|c|c|c|c|c|c|c|}
\hline \multicolumn{8}{|c|}{$\begin{array}{l}\text { TABLA 4.- DISTRIBUCION DEL DIÁMETRO DE LOS IMPLANTES SEGÚN } \\
\text { LAS TÉCNICAS QUIRÚRGICAS COMPLEJAS }\end{array}$} \\
\hline Técnicas & $3 \mathrm{~mm}$ & $3,3 \mathrm{~mm}$ & $3,45 \mathrm{~mm}$ & $3,75 \mathrm{~mm}$ & $3,8 \mathrm{~mm}$ & $4,2 \mathrm{~mm}$ & Total \\
\hline Injerto & - & 4 & 3 & 3 & 2 & - & 11 \\
\hline Expansión ósea & - & 4 & 4 & - & - & - & 8 \\
\hline Elevación seno maxilar & 1 & 3 & 1 & 22 & 29 & 21 & 74 \\
\hline Total & 1 & 11 & 8 & 25 & 29 & 19 & 93 \\
\hline
\end{tabular}


Un segundo requisito importante es la experiencia profesional en implantología oral. Aunque son técnicas complejas, pueden ser realizadas por un cirujano oral y/o maxilofacial o por un dentista general con suficiente entrenamiento quirúrgico en el campo de la implantología. Es evidente que la complejidad de la técnica aumenta desde una expansión ósea hasta una elevación traumática del seno maxilar o la realización de un injerto. En este sentido, cada vez son más frecuentes los procedimientos protocolizados que configuran estas técnicas complejas basados en la experiencia a largo plazo de grupos de trabajo o investigación ${ }^{15,20-22}$.

Un tercer requisito en el tratamiento con implantes oseointegrados del maxilar superior con técnicas quirúrgicas complejas es la utilización de implantes con diseños y superficies adecuadas ${ }^{23}$. En este sentido, los avances en el campo de la implantología han impulsado el desarrollo de implantes con unas características morfológicas que son especialmente recomendables para obtener una estabilidad primaria adecuada en el hueso del maxilar superior (frecuentemente pobre en calidad y cantidad) ${ }^{23-25}$. Además, la investigación en nuevas superficies rugosas (ej. chorreado de arena, grabado ácido, bioactivas) está mejorando la respuesta tisular incrementando el contacto hueso-titanio ${ }^{26-27}$. Estas dos características estructurales de los implantes deben ser utilizadas en la obtención de una favorable osteointegración, como es el caso del presente estudio donde se utilizaron implantes con una excelente capacidad autorroscante y una superficie rugosa con chorreado de arena y grabado ácido.

Un cuarto requisito para un buen éxito a largo plazo del tratamiento implantológico con técnicas quirúrgicas complejas del maxilar superior es la carga funcional y rehabilitación protésica ${ }^{28}$. En todos los tratamientos avanzados de cirugía ósea implantológica debe esperarse un tiempo mínimo de 6 meses para la carga funcional con el objetivo de que el tejido óseo esté suficientemente consolidado ${ }^{20,29}$. De esta forma, se puede proceder posteriormente a la restauración con prótesis fijas implantosoportadas (coronas y puentes) con resultados satisfactorios a largo plazo, como demuestra el presente estudio donde no hubo complicaciones tardías implantológicas que ocasionaran la pérdida de algún implante.

Los resultados clínicos del presente estudio indican que la realización de estas técnicas quirúrgicas pueden constituir un método muy favorable para el tratamiento implantológico del maxilar superior con edentulismo parcial y/o total con un grado más o menos avanzado de atrofia ósea, ya que la tasa de éxito del $96,8 \%$ confirma la elevada predecibilidad de esta alternativa terapéutica implantológica oral.

El uso de expansores para ensanchar el reborde alveolar superior representa un método sencillo para el dentista general que puede favorecer la inserción de implantes en crestas alveolares residuales estrechas con un éxito elevado ${ }^{30}$. En el presente estudio, el 15,6\% de los pacientes fueron tratados con expansores. La progresiva expansión del hueso mediante la utilización de los diversos expansores con diámetro creciente favorece una buena estabilidad primaria de los implantes (Figuras 1-5). En este sentido, un estudio español sobre 80 pacientes indica que no existen diferencias en el éxito largo plazo (2,7 años) de los implantes insertados de forma convencional (68 implantes y supervivencia del $92 \%$ ) y con expansores (158 implantes y una supervivencia del $90 \%$ ) en el maxilar superior, ya que los casos con fresado convencional eran más favorables en disponibilidad ósea que aquellos en los que se utilizaron expansores ${ }^{15}$.

En el presente estudio, en el 11,7\% de los pacientes se utilizaron injertos óseos para incrementar el volumen óseo y permitir la inserción correcta de los implantes. La reconstrucción de zonas maxilares edéntulas atróficas mediante la inserción de implantes y la realización de injertos óseos se está convirtiendo en una técnica rutinaria en la implantología oral ${ }^{20}$. Los resultados clínicos a 5-6 años, confirman que la realización de injertos óseos en maxilares superiores edéntulos $(75,1 \%)$ puede incrementar los fracasos de implantes comparados con los implantes insertados en maxilares sin injertos (84\%), probablemente debido a la escasa cantidad del hueso original, ya que la mayoría de los fracasos ocurrieron antes de la carga funcional ${ }^{31}$.

Sin embargo, otro estudio indica un elevado éxito de los implantes después de un periodo de más de 10 años de la realización de injertos en maxilares atróficos donde la supervivencia era superior al $90 \%{ }^{32}$. En este sentido, la inserción simultánea de los implantes con la realización del injerto en un mismo acto quirúrgico es una técnica poco invasiva para el paciente y acorta el tiempo real de tratamiento, no siendo necesaria una segunda intervención. No obstante, en aquellos casos con una mayor atrofia maxilar puede estar indicado la inserción posterior de los implantes con el objetivo de conseguir una mejor vascularización y solidez del injerto que facilite la estabilidad primaria de los implantes y minimice el riesgo de pérdida del injerto y de los implantes ${ }^{32}$. 
En el presente estudio, la mayor cantidad de pacientes tratados con técnicas complejas se correspondía con aquellos tratados mediante la elevación sinusal traumática $(74,5 \%)$ ya que la mayor parte de los pacientes presentaban un maxilar atrófico en la región posterior. Al contrario, los injertos y expansiones óseas se realizaron más frecuentemente en el sector anterior maxilar.

La elevación del seno maxilar es una técnica quirúrgica frecuentemente utilizada en implantología oral, aunque parece que la supervivencia de los implantes parece mostrar una mayor variabilidad en los senos injertados que en el maxilar posterior normal ${ }^{29}$. Una revisión reciente de la literatura indica que la tasa de supervivencia de los implantes en maxilares con elevaciones sinusales varía del 36 al 100\%, mientras que en áreas normales del maxilar posterior varía del $73 \%$ al $100 \%{ }^{33}$. En este sentido, la altura del hueso alveolar residual es citado como un factor pronóstico importante para el éxito de este tratamiento implantológico ${ }^{33}$.

Un estudio valora el tratamiento con implantes en 18 pacientes donde se realizaron 27 elevaciones sinusales con injertos óseos de procedencia, sobre todo, de la sínfisis mandibular. Después de un seguimiento medio de 162 semanas, el índice de supervivencia de los implantes era del 80,2\% lo que indica que esta técnica puede incrementar el volumen de hueso necesario para la inserción de los implantes pero la oseointegración y el éxito de los mismos están reducidos cuando se compara con los implantes insertados en el hueso maxilar normal ${ }^{34}$. De hecho en nuestro estudio, los implantes que fracasaron $(3,2 \%)$ fueron en aquellos pacientes a los que se realizó una elevación del seno maxilar.

En los casos de elevación sinusal del presente estudio se utilizaron como material de relleno el hueso autólogo del mismo paciente y hueso bovino mineralizado que ha sido valorado positivamente en algunos estudios ${ }^{35-36}$. Un amplia revisión que incluye 6.913 implantes insertados en 2.046 pacientes con elevaciones sinusales muestra una tasa global de supervivencia de los implantes del $91,4 \%$ (87,7\% solamente con injertos de hueso autólogo; $94,8 \%$ cuando se combina hueso autólogo con varios sustitutos óseos; y 95,9\% solamente con sustitutos óseos); lo que parece indicar que los sustitutos óseos son tan efectivos como el hueso autólogo usado solos o combinados ${ }^{37}$.

Las diversas técnicas quirúrgicas utilizadas en el presente estudio permiten aumentar el volumen óseo en sentido vertical (elevación del seno maxilar, injertos) u horizontal (expansión, injertos) pero no aseguran la oseointegración de los implantes. En este sentido, la pobre calidad del hueso alveolar atrófico del maxilar superior, sobre todo, en regiones posteriores no se modifica con estas técnicas y parece que puede ser un factor muy importante en estos casos ${ }^{34}$. De hecho, la mayor parte de los fracasos de los implantes en los diversos estudios ocurren durante el periodo de cicatrización o consolidación como en nuestro estudio donde los 3 implantes se perdieron de forma temprana y ninguno después de la rehabilitación protésica ${ }^{31,34}$.

El perfil sistémico del paciente es importante también para conseguir una buena oseointegración y un éxito a largo plazo del tratamiento con implantes ${ }^{16}$. En este sentido, desde el comienzo de la realización de protocolos sistemáticos en la implantología oral, la experiencia clínica ha demostrado una significativa relación entre el hábito de fumar y el fracaso de implantes ${ }^{38}$. En nuestro estudio, 2 de los 3 pacientes donde fracasaron los implantes eran fumadores. De hecho, el tabaco ha sido considerado un factor de riesgo para el tratamiento del maxilar atrófico mediante injertos y elevación sinusal como demuestra un estudio que revela que los fumadores (50\%) presentaban más complicaciones que los pacientes no fumadores $(23,1 \%)$. Además las complicaciones eran mayores entre los fumadores ${ }^{39}$.

La estabilidad primaria de los implantes insertados es un requisito esencial para conseguir una buena oseointegración. En este sentido las características estructurales son importantes porque el diseño, la longitud y el diámetro de los implantes pueden favorecer la estabilidad primaria ${ }^{25}$. Además de la cantidad y calidad del hueso, es determinante en el éxito de los implantes su diámetro y longitud como demuestra un estudio a 3 años donde la tasa de éxito era del 90,7\% en los implantes con un diámetro de 3-3,9 mm comparados con el 94,6\% para los implantes de diámetro 4$4,9 \mathrm{~mm}{ }^{40}$. Así mismo, la supervivencia fue del 66,7\% para los implantes de una longitud de $7 \mathrm{~mm}$ comparado con el $96,4 \%$ para los de $16 \mathrm{~mm}^{40}$.

Sin embargo, en nuestro estudio se utilizaron $15 \mathrm{im}-$ plantes de $10 \mathrm{~mm}$ sin ningún fracaso. Los implantes perdidos presentaban una mayor longitud $(11,5 \mathrm{~mm})$. Aunque existe una tendencia a considerar que los implantes más cortos fracasan más que los que presentan mayor longitud, la incorporación de nuevos diseños más autorroscantes y con superficies más rugosas como son los implantes utilizados en nuestro estudio 
que incrementan la estabilidad primaria están posibilitando que en muchas situaciones los implantes de longitud menor sea una alternativa implantológica sin un incremento en la tasa de fracasos ${ }^{25}$. En este sentido, las regiones posteriores del maxilar superior con un hueso escaso y pobre y que soportan grandes fuerzas oclusales representa un reto biológico importante para los implantes insertados. Sin embargo, un aumento del diámetro y buen tratamiento de la superficie (ej. mayor rugosidad) puede incrementar notablemente (300\%) el área de contacto hueso-implante, reducir el estrés y prevenir la pérdida del nivel de hueso y el fracaso prematuro de los implantes ${ }^{41}$.

\section{CONCLUSIONES}

El presente estudio demuestra la posibilidad de realización por el dentista general o cirujano oral y/o maxilofacial de las técnicas quirúrgicas complejas para mejorar las expectativas de tratamiento con implantes oseointegrados en el maxilar atrófico. Un correcto diagnóstico y planificación mediante un protocolo quirúrgico y prostodóncico adecuado con un periodo libre de carga funcional aceptable (más de 6 meses) y el uso de implantes con nuevos diseños microscópicos (ej. autorroscante) y microscópicos (ej. superficie tratada y rugosa) puede representar una alternativa con éxito en el tratamiento del paciente con edentulismo parcial o total superior.

\section{ABSTRACT}

Introduction. The aim of this study was to assess the clinical outcome of implant therapy of maxilla by advanced surgery techniques.

Methods. 51 patients were treated with 93 Microdent ${ }^{\circledR}$ sandblasted-etched surface implants for rehabilitation of maxilla by sinus floor augmentation, expansion technique and bone grafts. Implants were loaded after a healing free-loading period of 6 months with a followup at least of 24 months.

Results. 37 patients (72.5\%) were treated by sinus floor augmentation, 8 patients (15.6\%) were treated by expansion technique and 6 patients (11.7\%) with bone grafts. $83.3 \%$ of implants were inserted in posterior maxilla. Clinical findings showed a survival and success rate of implants of $96.8 \%$, because 3 implants were lost during the healing period.
Conclusions. This study indicate that treatment of maxilla by advanced surgery techniques with osseointegrated implants constitute a dental treatment with a high success rate success.

\section{KEY WORDS}

Oral implants, sinus floor elevation, bone graft, expansion technique, implant dentistry, maxilla.

\section{BIBLIOGRAFÍA}

1. Branemark PI, Hansson BO, Adell R, et al. Osseointegrated implants in the treatment of edentulous jaw. Experience for 10-years period. Scand J Plast Reconstr Surg 1977;11(Suppl 16):1-132.

2. Misch CE. Contemporary Implant Dentistry. San Luís: Mosby. 1999.

3. Cawood JL, Howell RA. A classification of the edentulous jaws. Int J Oral Maxillofac Surg 1988;17:232-6.

4. Lekholm U, Zarb GA. Selección y preparación del paciente. En: Branemark PI, Zarb GA, Albrektsson T. Prótesis tejido-integradas. La integración en la odontología clínica. Berlin: Quintessense 1987;pag: 199210 .

5. Takayama H. Consideraciones biomecánicas en los implantes osteointegrados. En: Hobo S, Ichida E, García LT. Osteointegración y rehabilitación oclusal. Madrid: Marban 1997;pag: 265-80.

6. Misch CE. Treatment planning for the edentulous posterior maxilla. En: Misch CE. Dental Implant Prosthetics. San Luís: Elsevier Mosby. 2005; pag: 265-80.

7. Peñarrocha M, Pi J. Situaciones especiales. En: Peñarrocha M. Implantología oral. Barcelona: Ars Médica. 2001;pag:95-126.

8. Becktor JP, Isaksson S, Sennerby L. Survival analysis of endosseous implants in grafted and nongrafted edentulous maxillae. Int J Oral Maxillofac Implants 2004;19: 107-15.

9. Aparicio C, Jensen O. Alveolar ridge wideninng by distraction osteogenesis: A case report. Pract Proced Aesthet Dent 2001;13:663-8. 
10. Tulasne JF. Osseintegrated fixtures in the pterigoid region. Advanced Ossointegration Surgery 1992;15: 182-8.

11. Aparicio C, Chantal M. El implante transzigomático. RCOE 1999;4:171-84.

12. Barrachina M. Acero J, De Paz V, Consejo C, Fernández A. Tratamiento del maxilar posterior atrófico mediante la técnica de elevación del seno maxilar y colocación de implantes dentales. RCOE 2002; 7:35-42.

13. Peñarrocha M, Guarinos J, Sanchis JM,. Balaguer J. Estudio clínico retrospectivo (1994-99) de 441 implantes ITI en 114 pacientes con un seguimiento medio de 2,3 años. Medicina Oral 2002; 7:144-55.

14. Donado M. Anatomía Implantológica. Bases morfológicas y su aplicación clínica en implantología dental. Barcelona: Ars Médica. 2003.

15. Peñarrocha M, Sanchis JM, Guarinos J, Soriano I, Balaguer J. Estudio comparativo entre la técnica convencional y la de osteodilatadores para la creación del lecho implantológico. A propósito de 226 implantes colocados en 80 pacientes. Av Periodoncia Impl Oral 2000;10:189-98.

16. Buser D, von Arx T, ten Bruggenkate C, Weingart D. Basic surgical principles with ITI implants. Clin Oral Impl Res 2000; 11 (suppl 1):59-68.

17. van Steenberghe D, Quirynen, Naert I. Survival and success rates with oral endosseous implants. En: Lang NP, Karring T, Lindhe J (eds.). Proceedings of the 3rd European Workshop on Periodontology. Implant Dentistry. Berlin: Quintessence. 1999; pag: 242-52.

18. Jacobs R. Preoperative radiologic planning of implant surgery in compromised patients. Periodontology 2000 2003;33:12-25.

19. Sanchis JM, Donado A, Peñarrocha M. Diagnóstico. En: Peñarrocha M. Implantología oral. Barcelona: Ars Médica. 2001; pag: 35-51.

20. Weingart D, Petrin G. Maxillofacial applications of implants in conjuction with bone grafts. En: Lang NP, Karring T, Lindhe J (eds.). Proceedings of the 3rd European Workshop on Periodontology. Implant Dentistry. Berlin: Quintessence. 1999; pag: 544-56.
21. Jensen OT, Shulman LB, Block MS, Iacono VJ. Report of the Sinus Consensus Conference of 1996. Int J Oral Maxillofac Implants 1998;13 (suppl).

22. Triplett RG, Schow SR, Laskin DM. Oral and maxillofacial surgery advances in implant dentistry. Int J Oral Maxillofac Implants 2000;15:47-55.

23. Binon PP. Implants and components : entering the new millennium. Int J Oral Maxillofac Implants 2000;15:76134.

24. Martinez H, Davarpanah M, Missika P, Celleti R, Lazzara R. Optimal implant stabilization in low density bone. Clin Oral Implant Res 2001;12:423-32.

25. Steigenga JT, Al-Shammani KF, Nociti FH, Misch CE, Wang HL. Dental implant design and its relationship to long-term implant success. Implant Dent 2003;12:306-17.

26. Khang W, Feldman S, Hawley CE, Gunsolley J. A multicenter study comparing dual acid-etched and machined-surfaced implants in various bone qualities. J Periodontol 2001;72:1384-90.

27. Trisi P, Lazzara R, Rebaudi A, Rao W, Testori T, Porter S. Bone-implant contact on machined and dual acidetched surfaces after 2 months of healing in the human maxilla. J Periodontol 2003; 74: 945-956.

28. Kim Y, Oh TJ, Misch CE, Wang HL. Occlusal considerations in implant therapy: clinical guidelines with biomechanical rationale. Clin Oral Implant Res 2005; 16:26-35.

29. Pérez O, Velasco E, García A, López J, Medel R. La elevación del seno maxilar en el tratamiento con implantes oseointegrados. Arch Odontoestomatol. En prensa.

30. Calvo JL, Sáez R, Pardo G, Carrión MJ. Ensachamiento del reborde alveolar posterior del maxilar superior con ostetomos compresivos. Estudio prospectivo a 9 meses. Rev Int Prot Estomatol 2005;7:272-8.

31. Becktor JP, Isaksson S, Sennerby L. Survival analysis of endosseous implants in grafted and nongrafted edentulous maxillae. Int J Oral Maxillofac Implants 2004; 19:107-15.

32. Yerit K, Posch M, Hainich S, Turhani D, Klug C, Wanschitz Fetal. Long-term implant survival in the grafted maxilla: results of a 12-year retrospective study. Clin Oral Impl Res 2004;15:693-9. 
33. Graziani F, Donos N, NeedlemanI, Gabriele M, Tonetti M. Comparisom of implant survival following sinus floor augmentation procedures with implants placed in pristine posterior maxilla bone: a systematic review. Clin Oral Impl Res 2004;15:677-82.

34. McCarthy C, Patel RR, Wragg PF, Brook IM. Sinus augmentation bone grafts for the provision of dental implants: report of clinical outcome. Int J Oral Maxillofac Implants 2003;18:377-82.

35. Yildirim M, SpiekermannH, Biesterfeld S, Edelhoff D. Maxillary sinus augmentation using xenogenic material (Bio-Oss) in combination with veinous blood: a histologic and histomorphometric study in humans. Clin Oral Impl Res 2000, 11:217-9.

36. Valentini P, Abensur DJ. Maxillary sinus grafting with anorganic bovine bone: a clinical report of long-term results. Int J Oral Maxillofac Implants 2003;18:556-60.

37. Del Fabbro M, Testori T, Francetti L, Weinstein R. Systematic review of survival rates for implants placed in the grafted maxillary sinus. Int J Periodontics Rest Dent 2004; 24:565-77.
38. McDermott NE, Chuang SK, Woo VV, Dodson TB. Complications of dental implants: identification, frequency, and associated risk factors. Int J Oral Maxillofac Implants 2003;18:848-55.

39. Levin L, Herzberg R, Dolev E, Schwartz-Arad D. Smoking and complications of onlay bone grafts and sinus lift operations. Int J Oral Maxillofac Implants 2004;19: 369-73.

40. Winkler S, Morris HF, Ochi S. Implant survival to 36 months as related to length and diameter. Ann Periodontol 2000;5:22-31.

41. Misch CE. Implant design considerations for the posterior regions of the mouth. Implant Dent 1999; 8:376-86.

\section{CORRESPONDENCIA}

Prof. Eugenio Velasco Ortega

Facultad de Odontología

$\mathrm{C} /$ Avicena, s/n.

41009 Sevilla

Tfno: 954481132

email: evelasco@us.es 